\title{
Addendum to: Assessment of Digital Image Correlation Measurement Errors: Methodology and Results [Experimental Mechanics 49(3)]
}

\author{
M. Bornert • F. Brémand • P. Doumalin • J.-C. Dupré • \\ M. Fazzini • M. Grédiac • F. Hild • S. Mistou • \\ J. Molimard • J.-J. Orteu • L. Robert • Y. Surrel • \\ P. Vacher $\cdot$ B. Wattrisse
}

The synthetic speckle images that were used to perform the simulations can be downloaded from the following website:

http://www.gdr2519.cnrs.fr/image_database/ ExpMech2009_vol49-3_pp353-370/

Workgroup "Metrology" of the French CNRS research network 2519 "Mesures de Champs et Identification en Mécanique des Solides / Fullfield measurements and identification in solid mechanics"

\section{Bornert}

Laboratoire Navier, École des Ponts ParisTech / IFSTTAR / CNRS

UMR 8205, Université Paris Est, 77455 Marne-la-Vallée Cedex,

France

F. Brémand · P. Doumalin · J.-C. Dupré

LMS, UMR 6610, Université de Poitiers, 86962 Futuroscope

Chasseneuil, France

M. Fazzini - S. Mistou

ENIT, Tarbes, France

\section{Grédiac}

Institut Pascal UMR CNRS 6602, Campus des Cézeaux, 24

avenue des Landais,

63174 Aubière, France

F. Hild

LMT-Cachan, ENS Cachan / CNRS / University Paris 6 / PRES

UniverSud Paris, Cachan, France

The readers interested in assessing the metrological performance of their own DIC software are invited to download these files, to use them as input data and to discuss matters related to their results with the authors.

\author{
J. Molimard \\ SMS, LTDS, UMR CNRS 5513, Mines Saint Étienne, Saint \\ Étienne, France \\ J.-J. Orteu $\cdot$ L. Robert $(\bowtie)$ \\ Université de Toulouse, Mines Albi, ICA (Institut Clément Ader), \\ 81013 Albi, France \\ e-mail: Laurent.Robert@mines-albi.fr \\ Y. Surrel \\ Visuol Technologies, Metz, France \\ P. Vacher \\ SYMME, Université de Savoie, Annecy, France \\ B. Wattrisse \\ LMGC, UMR CNRS 5508, Université Montpellier II, Montpellier, \\ France
}

